\title{
INSTABILITY OF A CONTINUOUSLY INHOMOGENEOUS VISCOELASTIC HALF-SPACE UNDER INITIAL STRESS
}

\author{
BY
}

M. A. BIOT 1

\begin{abstract}
Surface folding due to instability is analyzed for continuously inhomogeneous halfspace of viscoelastic properties. The medium is under the combined action of a horizontal compression and gravity. The material is of uniform density. Its viscoelastic properties vary exponentially with depth so that it degenerates into a fluid at large depth. It is assumed that the deformations verify the thermodynamic theory of irreversible processes. Folding wavelengths are evaluated. The general correspondence principle as formulated earlier by the author shows the results to be immediately applicable to either viscoelastic or purely elastic media. A numerical application to the geophysical scale indicates that a continuous viscosity gradient resulting from the temperature variation with depth cannot account for orogenesis by the mechanism of instability in compression.
\end{abstract}

\section{INTRODUCTION}

In previous work $(1,2,3)^{2}$ we have examined the stability of inhomogeneous viscoelastic media under initial stress. All these problems dealt with a discontinuous system in which one homogeneous layer was embedded in another homogeneous medium or lying on top of the latter. The influence of gravity was also examined (4). Part of the initial stress in addition to the hydrostatic pressure is a compressive stress which produces an instability. This instability manifests itself by the growth of folds in the layer. The surface instability of a homogeneous half-space was also treated (3) as a particular case of a more general problem.

The present analysis deals with a half-space whose inhomogeneity is continuously distributed. The hydrostatic pressure due to the weight of the material is superposed on a horizontal compression.

The material is viscoelastic and incompressible with properties decaying exponentially with depth so that the medium becomes a fluid at large depth. The physical properties assumed are quite general but subject to the requirements of the thermodynamics of irreversible processes (6). The analysis covers the particular limiting cases of the inhomogeneous purely viscous solid and the inhomogeneous purely elastic medium for which the instability degenerates into a buckling. Section 2

${ }^{1}$ Shell Development Company, Exploration and Production Research Division, Houston, Texas.

${ }^{2}$ The boldface numbers in parentheses refer to the references appended to this paper. 
presents the analytical formulation of the problem in which we have used equations for the deformation of a continuum under initial stress developed much earlier (7). Section 3 deals with the analytical solution and Section 4 with the numerical discussion. A numerical application to geophysics is also discussed indicating that large scale folding in the carth cannot be explained by the present mechanism on the basis of a continuous and isotropic viscosity gradient which itself is the result of the temperature increase with depth.

The results obtained here are equally applicable to either viscoelastic or purely elastic media. This is a consequence of the general correspondence principle as formulated and discussed by this writer in earlier work $(6,8,10)$. The plot in Fig. 2, for example, applies immediately to the case of a purely elastic half-space whose rigidity modulus decreases exponentially with depth, and the buckling stress is given by Eq. 41 .

Results of the experimental verification of the theory of folding of layered viscoelastic media by the use of models will be presented in later publications. A preliminary report on these tests has already been given (5). The task data show good agreement with the theory.

The author is indebted to A. Winzer for assistance in the analytical work and to A. S. Ginzbarg for the numerical solutions on the digital computer.

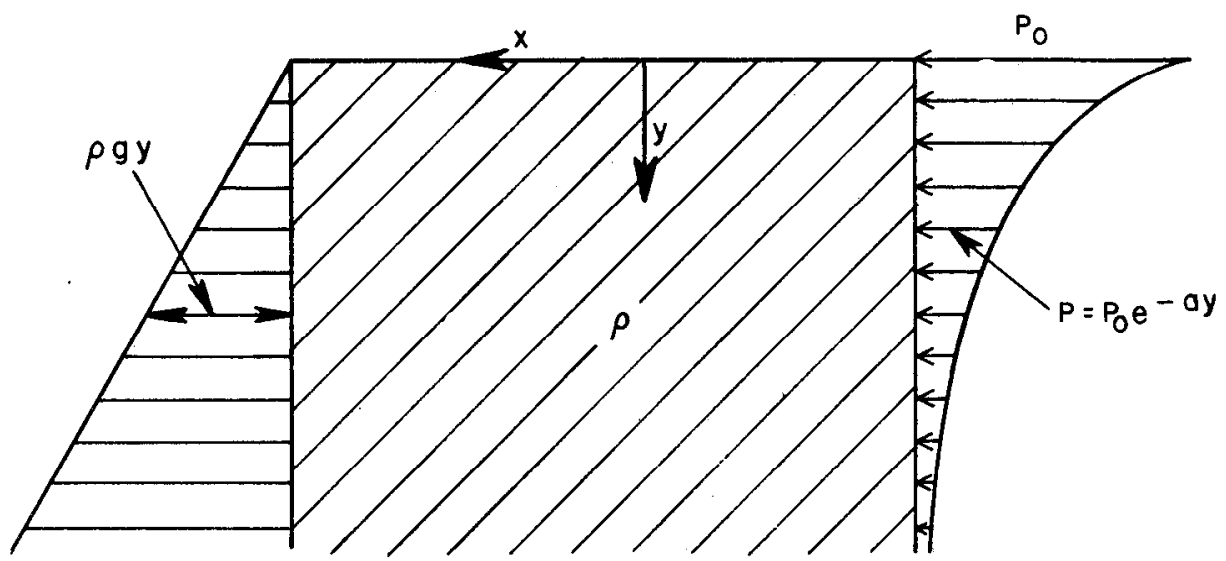

FIG. 1. Inhomogeneous viscoelastic half-space. The initial stress is the hydrostatic pressure $\rho g y$ and a horizontal compression $P$ function of depth.

\section{FORMULATION OF THE PROBLEM}

Consider an inhomogeneous viscoelastic half-space. We shall assume that it is incompressible. This assumption is not essential and the methods uscd hercafter are applicable to compressible media. However, the assumption simplifies the algebra quite appreciably. 
The $x$-axis lies along the surface of the half-space and the $y$-axis is directed downward (Fig. 1). Let this medium be in a state of initial stress which is the result of the gravity forces and a horizontal compression $P(y)$ function of the depth $y$. The state of initial stress is then represented by the components

$$
\begin{aligned}
& S_{11}=-P(y)-\rho g y \\
& S_{22}=-\rho g y \\
& S_{12}=0
\end{aligned}
$$

where $\rho$ is the mass density of the medium chosen to be constant and $g$ is the acceleration of gravity. For $P=0$ the initial stress reduces to a hydrostatic pressure, $S_{11}=S_{22}=-\rho g y$.

In the following, we have used the strain components

$$
\begin{aligned}
& e_{x x}=\frac{\partial u}{\partial x} \quad e_{y y}=\frac{\partial v}{\partial y} \\
& e_{x y}=\frac{1}{2}\left(\frac{\partial v}{\partial x}+\frac{\partial u}{\partial y}\right)
\end{aligned}
$$

and the rotation

$$
\omega=\frac{1}{2}\left(\frac{\partial v}{\partial x}-\frac{\partial u}{\partial y}\right)
$$

In these expressions, $u$ and $v$ denote the displacement components of the medium taken as zero in the state of initial stress. We now apply the general equations derived in previous work for the deformation of a prestressed medium (7). Equations for the prestressed viscoelastic medium were derived in (8) from a correspondence principle. For the particular state of prestress defined in Eqs. 1, the equations to be satisfied by the stress field are

$$
\begin{aligned}
& \frac{\partial s_{11}}{\partial x}+\frac{\partial s_{12}}{\partial y}+\rho g \omega-P \frac{\partial \omega}{\partial y}+\left(\frac{\partial P}{\partial y}+\rho g\right) e_{x y}=0 \\
& \frac{\partial s_{12}}{\partial x}+\frac{\partial s_{22}}{\partial y}-P \frac{\partial \omega}{\partial x}-\rho g e_{x x}=0 .
\end{aligned}
$$

The components $s_{11}, s_{22}$ and $s_{12}$ represent the incremental stress field with respect to axcs rotated locally through an angle $\omega$.

The physical properties of the material are represented by the stressstrain relations. With certain limitations which are discussed elsewhere $(2,5)$ and are not important in the present problem, the stressstrain relations for the incremented stresses are the same as for an 
incompressible material in the absence of pre-stress. They are written

$$
\begin{aligned}
s_{11}-s & =2 Q^{*} e_{x x} \\
s_{22}-s & =2 Q^{*} e_{y y} \\
s_{12} & =2 Q^{*} e_{x y} .
\end{aligned}
$$

The left-hand side represents the stress deviator, that is, we put

$$
\frac{1}{2}\left(s_{11}+s_{22}\right)=s .
$$

The operator $Q^{*}$ was derived (6) from the thermodynamics of irreversible processes and shown to be

$$
Q^{*}=\int_{0}^{\infty} \frac{p}{p+r} Q(r) d r+Q+Q^{\prime} p
$$

with $p=\frac{d}{d t}$. In the present problem the material is assumed inhomogeneous with properties function of the depth $y$. This will be expressed by making the quantities $Q(r), Q$ and $Q^{\prime}$ functions of $y$ in the operator $Q^{*}$.

The boundary condition results from the absence of forces on the free surface $(y=0)$. Using expressions for the boundary forces established in the earlier publications $(3,7)$ the boundary conditions at $y=0$ are expressed as

$$
s_{22}=0 \quad e_{x y}=0 .
$$

The derivation of these boundary conditions follows very closely that in (3) and (4).

The problem will be further particularized by assuming viscoelastic properties represented by the operator

with

$$
Q^{*}=Q_{0}^{*} e^{-a y}
$$

$$
Q_{0}{ }^{*}=\int_{0}^{\infty} \frac{p}{p+r} Q(r) d r+Q+Q^{\prime} p
$$

This last operator is independent of $y$, and the inhomogeneity is represented only by the exponential factor in $Q^{*}$. This corresponds to viscoelastic properties which are nonhomogeneous but such that the relaxation spectrum is independent of the location. We used the term "homogeneous spectrum" to describe this type of viscoelasticity and have shown that it is associatcd with special propertics as regards the stress distribution $(8,9)$.

In a material represented by the operator (Eq. 9) the pre-stress 
must also follow a similar law (Fig. 1). We write

$$
P=P_{0} \epsilon^{-a y} \text {. }
$$

Mathematically the problem reduces to solving Eqs. 4 after substituting the values of Eqs. 5 for the stresses. These are two equations for the three unknowns $u, v$, and $s$. A third equation is furnished by the condition of incompressibility.

$$
\frac{\partial u}{\partial x}+\frac{\partial v}{\partial y}=0
$$

The boundary conditions at $y=0$ are given by Fqs. 8 and a further condition is that the solution must vanish at $y=\infty$.

\section{ANALYTICAL SOLUTION}

Following a procedure already used (4), it is possible to simplify the problem by eliminating gravity from the differential equations, provided the boundary conditions are appropriately modified. This is done by replacing the stress $s_{i}$ by $s_{i j}-\rho v g \delta_{i j .}{ }^{3}$ This substitution does not affect the stress-strain relations of Eqs. 5. On the other hand, if we take into account the condition of incompressibility (Eq. 12), Eqs. 4 become

$$
\begin{array}{r}
\frac{\partial s_{11}}{\partial x}+\frac{\partial s_{12}}{\partial y}-P \frac{\partial \omega}{\partial y}+\frac{\partial P}{\partial y} \epsilon_{x y}=0 \\
\frac{\partial s_{12}}{\partial x}+\frac{\partial s_{22}}{\partial y}-P \frac{\partial \omega}{\partial x}=0
\end{array}
$$

The boundary conditions (Eqs. 8) are replaced by

$$
\begin{aligned}
& s_{22}=\rho g v \\
& e_{x y}=0 .
\end{aligned}
$$

This is a particular application of a quite general procedure by which the hydrostatic stress is eliminated from the problem while a "buoyancy" term is added at the boundary.

The condition of incompressibility is satisfied by putting

$$
\begin{aligned}
& u=\frac{\partial \phi}{\partial y} \\
& v=-\frac{\partial \phi}{\partial x} .
\end{aligned}
$$

\footnotetext{
${ }^{3} \delta_{i j}$ is the Kronecker symbol, that is, $\delta_{11}=\delta_{22}=1$ and $\delta_{12}=0$.
} 
By substitution of the stresses (Eqs. 5) into Eqs. 13 and using expressions (15) for the displacements $u$ and $v$, we arrive at two equations for the two unknowns $\phi$ and $s$. We further particularize the solution by looking for deformations which are sinusoidal along $x$. We therefore put

$$
\begin{aligned}
& \phi=\varphi(y) \sin l x \\
& s=P_{0} f(y) \epsilon^{-a z} \cos l x .
\end{aligned}
$$

The unknown functions $\varphi$ and $f$ must satisfy the ordinary differential equations

$$
\begin{aligned}
& \frac{1}{2} \frac{1+\zeta}{\zeta}\left(\varphi^{\prime \prime \prime}-\varphi^{\prime} l^{2}-a \varphi^{\prime \prime}-a \varphi l^{2}\right)-l f=0 \\
& \frac{1}{2} \frac{1-\zeta}{\zeta}\left(\varphi l^{2}-\varphi^{\prime \prime}\right) l+\frac{1}{\zeta} a \varphi^{\prime} l+f^{\prime}-a f=0
\end{aligned}
$$

The primes represent derivatives with respect to $y$ and we have put

$$
\zeta=\frac{P_{0}}{2 Q_{0}^{*}}
$$

Solutions of Eqs. 17 arc

$$
\begin{aligned}
& \varphi=A e^{\beta_{1} a y}+B e^{\beta_{2} a y} \\
& f=C e^{\beta_{1} a y}+D e^{\beta_{2} a y} .
\end{aligned}
$$

Substitution of these expressions into Eqs. 17 leads to the characteristic equation for $\beta$,

$$
\left[\beta\left(\beta^{2}-\delta^{2}\right)-\left(\beta^{2}+\delta^{2}\right)\right](\beta-1)+k^{2} \delta^{2}\left(\delta^{2}-\beta^{2}\right)+\frac{2 \beta \delta^{2}}{1+\zeta}=0
$$

where

$$
\begin{aligned}
& k^{2}=\frac{1-\zeta}{1+\zeta} \\
& \delta=l / a .
\end{aligned}
$$

There are two roots, $\beta_{1}$ and $\beta_{2}$, for $\beta$ which have negative real parts. These two roots are used in the exponents of the solution expressed by Eqs. 19. This insures that the solution vanishes for $y=\infty$ (that is, at infinite depth). Equation 20 is a fourth degree equation in $\beta$. However, it is easily solved by noticing that it may be written as a second degree equation in $\beta(\beta-1)$; for example, we may write

$$
\zeta=\frac{\delta^{4}-\delta^{2}[2 \beta(\beta-1)-1]+[\beta(\beta-1)]^{2}}{\delta^{4}-\delta^{2}-[\beta(\beta-1)]^{2}} .
$$


Solving this quadratic equation for $\beta(\beta-1)$ and again a second quadratic equation for $\beta$, we find

$$
\left.\begin{array}{c}
\beta_{1} \\
\beta_{2}
\end{array}\right\}=\frac{1}{2}\left[1-\sqrt{1+\frac{4}{1+\zeta}\left(\delta \pm \sqrt{\left.\delta^{2} \zeta^{2}-(1+\zeta)^{2}\right)}\right.}\right] .
$$

In the limiting case for $\delta \rightarrow \infty$, the roots reduce to

$$
\begin{aligned}
& \beta_{1}=-\delta=-l / a \\
& \beta_{2}=-k \delta=-k l / a .
\end{aligned}
$$

This case corresponds either to $a=0$, that is, to the homogeneous halfspace already examined (3) or to $l \rightarrow \infty$, that is, to a vanishing wavelength.

The four constants $A, B, C, D$ in Eqs. 19 are not arbitrary. The relations among them are found by substitution in the differential equations. There are, therefore, only two arbitrary constants.

These two remaining constants are now eliminated by taking into account the boundary condition (Eq. 14) at the free surface $(y=0)$. This leads to the following characteristic equation:

$$
\zeta=\frac{2 \delta^{2}\left[\delta^{2}-\beta_{1} \beta_{2}-G\left(\beta_{1}+\beta_{2}\right)\right]}{\beta_{1}^{2} \beta_{2}^{2}-\delta^{4}+\delta^{2}\left(\beta_{1}+\beta_{2}\right)\left(\beta_{1}+\beta_{2}-2 G\right)}-1 .
$$

The parameter

$$
G=\frac{\rho g}{P_{0} a}
$$

represents the influence of gravity in non-dimensional form.

\section{NUMERTCAL RESULTS}

The characteristic equation (24) may be considered as a relation between $\zeta$ and $\delta$ where $G$ plays the role of a parameter. For a given value of $G$, this relationship represents the instability of the system as a function of the wavelength. This can easily be seen if we go back to the significance of the operator $p$ as discussed in our earlier treatment of similar problems. A certain value of $\zeta$ corresponds to a certain positive value of $p$ for which a time-dependent solution exists and is proportional to the increasing exponential $e^{p t}$. The value of $p$ contained in $\zeta$ is a measure of the instability.

An important property may be stated here which is dependent on the nature of the operator $Q_{0}{ }^{*}$ itself. We have derived its form (Eq. 10) from the thermodynamics of irreversible processes. As pointed out in (6) and later papers, a consequence of the thermodynamics is the positive sign of all the terms in Eq. 10 . Hence $Q_{0}{ }^{*}$ is a monotonically in- 
creasing function of $p$. Therefore, considering the relationship between $\zeta$ and $\delta$, a minimum of $\zeta$ corresponds to a maximum of $p$. For this value of $\delta$ the rate of growth of the deformation is a maximum. Wavelengths corresponding to this case will tend to appear more strongly than others. Following the terminology introduced in the earlier work we shall call this wavelength the dominant wavelength.

In solving the characteristic equation (24), we must distinguish cases for which the roots $\beta_{1}$ and $\beta_{2}$ are real or complex. From Eq. 22 it can be seen that real roots occur in the region

$$
\delta>\frac{1}{\zeta}+1
$$

and complex roots in the region defined by changing the direction of the inequality. For the case of complex roots, calculations are conveniently carried out by putting

$$
\begin{aligned}
R & =\left[1+20 \delta^{2}+4 \delta^{2}\left(1+4 \delta^{2}\right)\left(\frac{1-\zeta}{1+\zeta}\right)\right]^{1 / 4} \\
\cos \frac{\theta}{2} & =\frac{1}{\sqrt{2}}\left[1+\frac{1}{R^{2}}\left(1+\frac{4 \delta^{2}}{1+\zeta}\right)\right]^{1 / 2} \\
\beta_{1}+\beta_{2} & =1-R \cos \frac{\theta}{2} \\
\beta_{1} \beta_{2} & =\frac{1}{4}\left[1+R^{2}-2 R \cos \frac{\theta}{2}\right] .
\end{aligned}
$$

The complex roots themselves are

$$
\begin{aligned}
& \beta_{1}=\frac{1}{2}\left(1-R \cos \frac{1}{2} \theta+i R \sin \frac{1}{2} \theta\right) \\
& \beta_{2}=\frac{1}{2}\left(1-R \cos \frac{1}{2} \theta-i R \sin \frac{1}{2} \theta\right) .
\end{aligned}
$$

The characteristic equation (Eq. 24) was solved on an automatic computer for five values of the parameter $G$

$$
G=0, \frac{1}{10}, \frac{1}{25}, \frac{1}{60}, \frac{1}{250} .
$$

Plots of $\zeta$ versus $\delta$ are shown in Fig. 2 .

The diagrams are plotted in the range $\delta<1$. In this range the roots $\beta_{1}$ and $\beta_{2}$ are complex. The minimum value of $\zeta$ and the corresponding values of $\delta$ and $\beta_{1}, \beta_{2}$ are given in Table $I$.

The value $\delta_{d}$ of $\delta$ which corresponds to $\zeta_{\mathrm{min}}$ yields the dominant wavelength, 


$$
L_{d}=\frac{2 \pi}{a \delta_{d}}
$$

The value of $\delta_{d}$ for $G=1 / 1000$ was evaluated by interpolation. In the absence of gravity $(G=0)$, the dominant wavelength goes to infinity $\left(\delta_{d}=0\right)$. It is found that $\delta_{d}$ is represented approximately by the formula :4

$$
\delta_{d}=2.2 G^{6 / 10}
$$

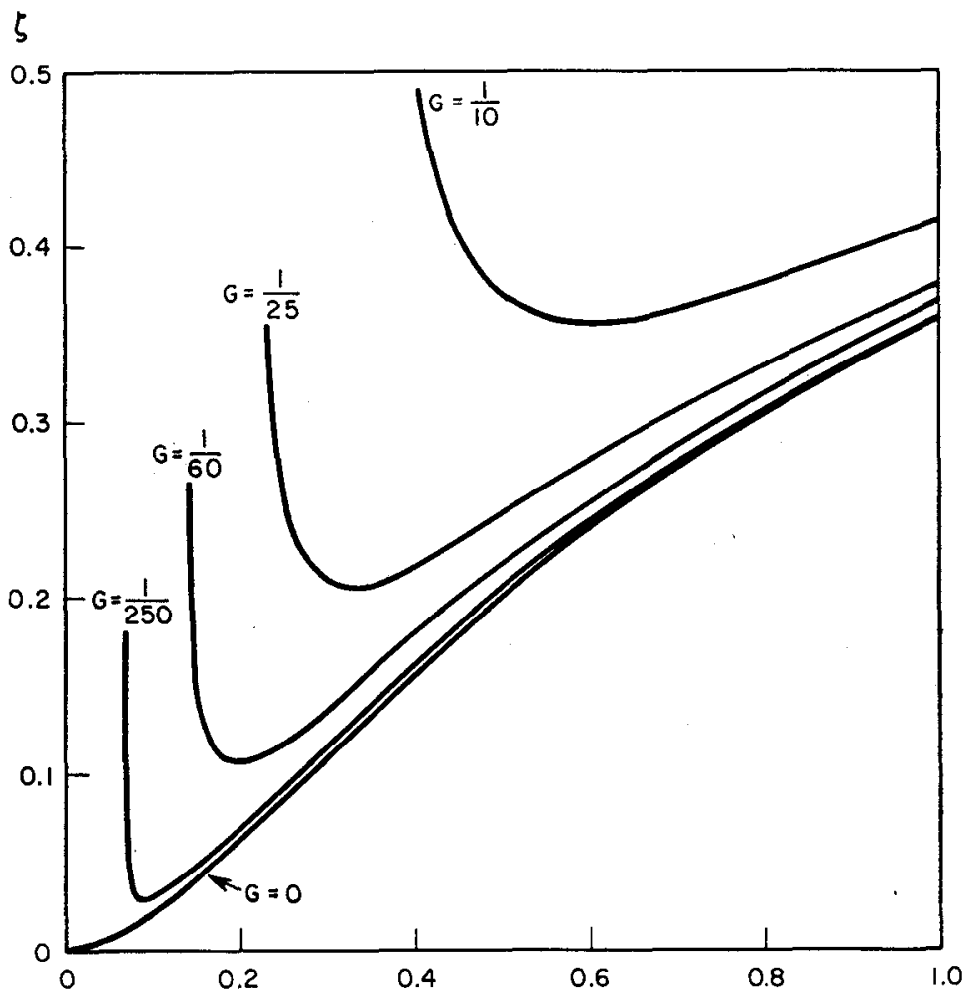

FIG. 2. The stability variable $\zeta$ as a function of the dimensionless wave-number $\delta$ and the gravity parameter $G$.

TABLE I.

$\begin{array}{cccc}G & \zeta_{\min } & \delta_{d} & \beta_{1}, \beta_{2} \\ 1 / 10 & 0.356 & 0.585 & -0.29 \pm 0.36 i \\ 1 / 25 & 0.206 & 0.326 & -0.13 \pm 0.35 i \\ 1 / 60 & 0.107 & 0.196 & -0.06 \pm 0.17 i \\ 1 / 250 & 0.029 & 0.074 & -0.010 \pm 0.072 i \\ 1 / 1000 & \ldots & 0.035 & \ldots \\ 0 & 0 & 0 & \ldots\end{array}$


The value of $\zeta$ goes to infinity below a certain value of $\delta$ which depends on $G$. The physical significance of this corresponds to the existence of a cut-off wavelength above which no instability exists. The value of $\delta$ for this vertical asymptote is determined by the condition that the denominator in Eq. 24 vanishes. For vanishingly small $\delta$, the location of the asymptote is given by

$$
\delta=\sqrt{G} .
$$

The limiting case of a vanishing wavelength $(l \rightarrow \infty)$ is found by putting $\delta=\infty$ in Eq. 24. This equation becomes

$$
\zeta^{3}+2 \zeta^{2}-2=0 .
$$

The real positive root of this equation is

$$
\zeta=0.839 \text {. }
$$

Hence the curves in Fig. 2 all tend toward the same horizontal asymptote for $\delta \rightarrow \infty$, that is, for vanishing wavelength. In this limiting case the influence of gravity disappears and the value of $\zeta$ (Eq. 34) is the same as that obtained previously (3) for the case of an homogeneous halfspace without gravity.

An interesting feature results from the complex nature of the roots $\beta_{1}$, $\beta_{2}$. This means that the displacements of the semi-infinite medium exhibit a damped-oscillatory character as a function of the depth $y$. This can be seen from Eqs. 19, which are linear combinations of functions of the type

$$
\exp \left[a y \operatorname{Re} \beta_{1}\right] \underset{\cos }{\sin }\left[a y \operatorname{Im} \beta_{1}\right]
$$

The real and imaginary parts $\operatorname{Re} \beta_{1}$ and $\operatorname{Im} \beta_{1}$ of $\beta_{1}$ are shown in Table I. For example, for $G=1 / 250$ the distribution with depth becomes

$$
\exp [-0.01 a y]_{\cos }^{\sin }[0.072 a y] \text {. }
$$

Over one wavelength the amplitude decays by a factor 0.418 . The values of Table I indicate that the decay decreases with decreasing values of $G$. In solving the problem no restriction has been put on the nature of the operator $Q_{0}{ }^{*}$ except that it satisfies thermodynamic principles. This insures the existence of one positive root $p$ corresponding to any value of $\zeta$ derived from the stability diagram (Fig. 2). In particular, the theory is applicable to a purely viscous medium. In this case the operator is

$$
Q_{0}^{*}=\eta_{0} p
$$


The viscosity $\eta$ of this medium decreases exponentially with depth according to the equation

$$
\eta=\eta_{0} e^{-a \nu} .
$$

The theory also applies to a purely elastic material. In this case

$$
Q_{0}{ }^{*}=\mu_{0} .
$$

The medium is an inhomogeneous elastic half-space whose rigidity modulus $\mu$ varies with depth according to the equation

$$
\mu=\mu_{0} e^{-a y} .
$$

The minimum value $\zeta_{\mathrm{min}}$ yields the buckling load $P_{0}$ by

$$
P_{0}=2 \mu_{0} \zeta_{\text {min }} \text {. }
$$

The dominant wavelength does not depend on the particular nature of the viscoelastic material. For instance, its value for the viscous solid is the same as the buckling wavelength for the elastic case. The difference lies in the fact that folding always occurs in the purely viscous case in contrast with the elastic case where a minimum compression (Eq. 41) is required for buckling to appear.

In applications it is of interest to consider the magnitude of the folding. As pointed out above, the amplification of waves after a time $t$ is $e^{p t}$. If we assume a viscous medium, the time required for it to shorten by 25 per cent at the surface is

$$
t_{1}=\frac{\eta_{0}}{P_{0}}
$$

and the amplification of any initial wave at that time is $e^{p t_{1}}$. If we want this amplification to be equal to 100 then we must have

$$
p t_{1}=\log _{e} 100=4.60 .
$$

Substituting for $p$ its expression in terms of $\zeta$ by eqs. 18 and 37 , we derive

$$
\zeta=\frac{1}{9.2}
$$

Hence this is the value of $\zeta$ required for the amplification to be 100 during a compressive deformation of 25 per cent. We see from Table I that for the dominant wavelength this requires that the value of $G$ be about

$$
G=\frac{1}{60}
$$


Smaller values of $G$ will generate higher amplifications. In application to geophysics we may put $P_{0}$ equal to a value of the order of the crushing strength of rock at the surface, for example, $P_{0}=10^{9}$ dynes $/ \mathrm{cm}^{2}$. With $\rho=2.5$ and $G=1 / 60$ we derive

$$
\frac{1}{a} \cong 65 \text { meters. }
$$

This is the depth in which the viscosity decreases by a factor $1 / e$.

The dominant wavelength for this case is found from Table I and Eq. 30. Its value is $L_{d}=2100$ meters. The viscosity gradient required for such significant folding to happen and which is characterized by the value (46) of $1 / a$ is much higher than that which results from increases of temperature with depth in the earth.

\section{REFERENCES}

(1) M. A. BIot, "Folding Instability of a Layered Viscoelastic Medium under Compression," Proc. Royal Soc. A, Vol. 242, pp. 444-454 (1957).

(2) M. A. Bтот, "On the Instability and Folding Deformation of a Layered Viscuelastic Medium in Compression," J. Appl. Mech., Vol. 27, pp. 393-400 (1959).

(3) M. A. Віот, "Folding of a Layered Viscoelastic Medium Derived from an Exact Stability Theory of a Continuum under Initial Stress," Quart. Appl. Math., Vol. 17, No. 2, pp. 185-204 (1959).

(4) M. A. Віот, "The Influence of Gravity on the Folding of a Layered Viscoelastic Medium under Compression," Jour. Frankuin Inst., Vol. 267, pp. 211-227 (1959).

(5) M. A. Bıот, "Stability Problems of Inhomogeneous Viscoelastic Media," Proc. IUTAM Colloquium on Non-homogeneity in Elasticity and Plasticity Warsaw, September 1958. London, Pergamon Press, 1959.

(6) M. A. Bıot, "Theory of Stress Strain Relations in Anisotropic Viscoelasticity and Relaxation Phenomena," J. Appl. Phys., Vol. 25, pp. 1385-1391 (1954).

(7) M. A. Bıot, "Nonlinear Theory of Elasticity and the Linearized Case for a Body under Initial Stress," Phil. Mag., Ser. 7, Vol. XXVII, pp. 468-489 (1939).

(8) M. A. Brot, "Variational and Lagrangian Methods in Viscoelasticity," Proc. IUTAM Colloquium on the Deformation and Flow of Solids (Madrid 1955), Berlin, Springer, 1956.

(9) M. A. Bior, "Linear Thermodynamics and the Mechanics of Solids," Proc. Third U. S. Nat. Cong. of Appl. Mech. Brown University, Published by ASME, 1958.

(10) M. A. BIot, "Dynamics of Viscoelastic Anisotropic Media," Proc. Fourth Midwestern Conference on Solid Mechanics, Purdue University, Sept., 1955. 
Equeation 22 meispeint.

$$
\frac{48}{1+5} \leftarrow
$$

